\title{
Toeplitz Algebras and Rieffel Deformations
}

\author{
L. A. Coburn, Jingbo Xia \\ Department of Mathematics, State University of New York, Buffalo, NY 14214
}

Received: 20 September 1993/in revised form: 7 May 1994

\begin{abstract}
We establish a representation theorem for Toeplitz operators on the SegalBargmann (Fock) space of $\mathbf{C}^{n}$ whose "symbols" have uniform radial limits. As an application of this result, we show that Toeplitz algebras on the open ball in $\mathbf{C}^{n}$ are "strict deformation quantizations", in the sense of M. Rieffel, of the continuous functions on the corresponding closed ball.
\end{abstract}

\section{Introduction}

In [R], Rieffel proposed a general scheme for producing "strict deformation quantizations" of $C^{*}$-algebras with $\mathbf{R}^{2 n}$ action. His scheme is modelled on classical Weyl quantization. As one example, Rieffel showed, following earlier work of Sheu [S], that the Toeplitz algebra $\tau(\mathbf{D})$ on the unit disc $\mathbf{D}$ arises from his scheme as a strict deformation quantization of the sup norm algebra $C(\mathbf{D})$ of continuous functions on the closed unit disc. In this note, we extend Rieffel's analysis to show that the Toeplitz algebra $\tau\left(\mathbf{B}_{2 n}\right)$ of the unit ball $\mathbf{B}_{2 n}$ (in $\mathbf{C}^{n}$ ) is a strict deformation quantization of the algebra $C\left(\mathbf{B}_{2 n}\right)$ of continuous functions on the closed unit ball.

Let $\mathbf{C}^{n}$ be the vector space of $n$-tuples of complex numbers with elements $z=\left(z_{1}, \ldots, z_{n}\right)$ and the usual norm $|z|=\left(\left|z_{1}\right|^{2}+\cdots+\left|z_{n}\right|^{2}\right)^{1 / 2}$. We denote by $\mathbf{B}_{2 n}$ the (real) $2 n$-dimensional open unit ball in $\mathbf{C}^{n}, \mathbf{B}_{2 n}=\left\{z \in \mathbf{C}^{n}:|z|<1\right\}$, and write $S^{2 n-1}=\left\{z \in \mathbf{C}^{n}:|z|=1\right\}$ for the unit sphere with $\overline{\mathbf{B}}_{2 n}=\mathbf{B}_{2 n} \cup S^{2 n-1}$.

In what follows, we consider three related Hilbert spaces of functions on $\mathbf{C}^{n}$. The first is the Bergmann space of Lebesgue volume $(d v)$-square-integrable holomorphic functions on the open unit ball $\mathbf{B}_{2 n}, H^{2}\left(\mathbf{B}_{2 n}\right)$. The next, is the space of Lebesgue surface area $(d \sigma)$-square-integrable functions on the unit sphere $S^{2 n-1}$ which extend to be holomorphic in $\mathbf{B}_{2 n}, H^{2}\left(S^{2 n-1}\right)$. Finally we have the Segal-Bargmann space $H^{2}\left(\mathbf{C}^{n}\right)$ of entire functions on $\mathbf{C}^{n}$ which are square integrable with respect to the Gaussian measure $d \mu(z)=e^{-|z|^{2} / 2}(2 \pi)^{-n} d v(z)$. Here $d v$ and $d \sigma$ are normalized by $v\left(\mathbf{B}_{2 n}\right)=\pi^{n} / n$ ! and $\sigma\left(S^{2 n-1}\right)=2 \pi^{n} /(n-1)$ !. 
These spaces have the common feature that an orthonormal basis for each can be constructed in the form

$$
a_{k} z^{k}
$$

where $k=\left(k_{1}, \ldots, k_{n}\right)$ and $k_{j}$ are integers, $k_{j} \geqq 0$. Here $a_{k}$ is some complex scalar and

$$
z^{k} \equiv z_{1}^{k_{1}} z_{2}^{k_{2}} \ldots z_{n}^{k_{n}}
$$

is the standard monomial. Of course, the weights $a_{k}$ vary, depending on the space of functions. It is known $[\mathrm{C} 1, \mathrm{BC} 1]$ that on $H^{2}\left(\mathbf{B}_{2 n}\right)$, we have the orthonormal basis

$$
\tilde{e}_{k}=\left(\pi^{n}\right)^{-1 / 2}\left\{\frac{(|k|+n) !}{k !}\right\}^{1 / 2} z^{k},
$$

while on $H^{2}\left(\mathbf{C}^{n}\right)$, we have the orthonormal basis

$$
e_{k}=\left(2^{|k|} k !\right)^{-1 / 2} z^{k}
$$

where $|k| \equiv k_{1}+k_{2}+\cdots+k_{n}$ and $k ! \equiv k_{1} ! k_{2} ! \ldots k_{n} !$.

Our key technical result is that the canonical isometry $V$ from $H^{2}\left(\mathbf{B}_{2 n}\right)$ to $H^{2}\left(\mathbf{C}^{n}\right)$ defined by

$$
V \tilde{e}_{k}=e_{k}
$$

induces a representation in $\tau\left(\mathbf{B}_{2 n}\right)$ of Toeplitz operators on $H^{2}\left(\mathbf{C}^{n}\right)$ whose "symbols" have uniform radial limits. A related result, on Toeplitz operators whose symbols vary in the radial direction only, was obtained in [G, Theorem 10.1]. See also [H].

\section{Representation of Toeplitz Operators on $H^{2}\left(C^{n}\right)$}

In [C1, BC1], Toeplitz operators on $H^{2}\left(S^{2 n-1}\right), H^{2}\left(\mathbf{B}_{2 n}\right), H^{2}\left(\mathbf{C}^{n}\right)$ are defined and studied. For $f$ a bounded measurable function on the underlying space, the Toeplitz operator $T_{f}\left(\tilde{T}_{f}\right)$ is given by

$$
T_{f} g=P(f \cdot g),
$$

where $P$ is the orthogonal projection from $L^{2}$ onto the corresponding $H^{2}$ space. There are natural isometries from $H^{2}\left(S^{2 n-1}\right)$ to $H^{2}\left(\mathbf{B}_{2 n}\right)$ and from $H^{2}\left(\mathbf{B}_{2 n}\right)$ onto $H^{2}\left(\mathbf{C}^{n}\right)$ which map $\tilde{e}_{k} \rightarrow e_{k}$. In [C1, Theorem 1], it was shown that the natural isometry from $H^{2}\left(S^{2 n-1}\right)$ to $H^{2}\left(\mathbf{B}_{2 n}\right)$ "intertwines" Toeplitz operators in a suitably weak sense. Here, we consider the corresponding problem for $H^{2}\left(\mathbf{C}^{n}\right)$.

For $f$ a bounded measurable function on $\mathbf{B}_{2 n}$, we write $\tilde{T}_{f}$ for the Toeplitz operator on $H^{2}\left(\mathbf{B}_{2 n}\right)$. Similarly, for $f$ bounded measurable on $\mathbf{C}^{n}$, we write $T_{f}$ for the Toeplitz operator on $H^{2}\left(\mathbf{C}^{n}\right)$.

Key Lemma 1. The operators $T_{z_{J} /|z|}-V \tilde{T}_{z_{J}} V^{-1}$ are compact for all $j, 1 \leqq j \leqq n$.

Proof. By symmetry, it suffices to consider $j=1$. Direct calculation shows that

$$
\begin{gathered}
\tilde{T}_{z_{1}} \tilde{e}_{k}=\beta_{k} \tilde{e}_{k+\delta_{1}}, \\
T_{z_{1} /|z|} e_{k}=\alpha_{k} e_{k+\delta_{1}},
\end{gathered}
$$


where $\delta_{1}=(1,0,0, \ldots, 0)$. It follows that

$$
T_{z_{1} /|z|}-V \tilde{T}_{z_{1}} V^{-1}=S_{\delta_{1}} D
$$

where

$$
S_{\delta_{1}} e_{k}=e_{k+\delta_{1}}
$$

and

$$
D e_{k}=\left(\alpha_{k}-\beta_{k}\right) e_{k} .
$$

Thus, it will suffice to check that $D$ is compact, i.e. that for arbitrary $\varepsilon>0$,

$$
\left|\alpha_{k}-\beta_{k}\right|<\varepsilon
$$

for $k$ outside of some finite set of multi-indices $F_{\varepsilon}$.

We need very precise estimates on $\alpha_{k}-\beta_{k}$. It is not hard to check (as in [C1]) that

$$
\beta_{k}=\left(k_{1}+1\right)^{1 / 2}(|k|+n+1)^{-1 / 2} .
$$

The calculation of a useable value of $\alpha_{k}$ is more complicated. Direct calculation shows that

$$
\alpha_{k}=\left\{2\left(k_{1}+1\right)\right\}^{-1 / 2}\left(2^{|k|} k !\right)^{-1} \mathscr{I}
$$

where

$$
\mathscr{I}=\int_{0}^{\infty} \cdots \int_{0}^{\infty} \frac{r_{1}^{2\left(k_{1}+1\right)+1} r_{2}^{2 k_{2}+1} \ldots r_{n}^{2 k_{n}+1}}{\sqrt{r_{1}^{2}+\cdots+r_{n}^{2}}} e^{-\left(r_{1}^{2}+\cdots+r_{n}^{2}\right) / 2} d r_{1} \ldots d r_{n} .
$$

Making a change of variables in the first two coordinates to polar form, and proceeding inductively, we obtain

$$
\mathscr{I}=\int_{0}^{\infty} s^{2(|k|+n)} e^{-s^{2} / 2} d s \cdot \prod_{m=1}^{n-1} \int_{0}^{\pi / 2} \cos ^{2\left(k_{1}+\cdots+k_{m}+m\right)+1} \theta \sin ^{2 k_{m+1}+1} \theta d \theta
$$

It is a standard calculation $[\mathrm{BC} 1]$ that

$$
\int_{0}^{\infty} s^{2(|k|+n)} e^{-s^{2} / 2} d s=\frac{\{2(|k|+n)\} ! \sqrt{\pi}}{2^{|k|+n}(|k|+n) ! \sqrt{2}} .
$$

A beautiful classical result of Euler $[\mathrm{WW}]$ is that

$$
\int_{0}^{\pi / 2} \cos ^{2 m_{1}+1} \theta \sin ^{2 m_{2}+1} \theta d \theta=\frac{1}{2} \frac{m_{1} ! m_{2} !}{\left(m_{1}+m_{2}+1\right) !} .
$$

It follows that

$$
\prod_{m=1}^{n-1} \int_{0}^{\pi / 2} \cos ^{2\left(k_{1}+\cdots+k_{m}+m\right)+1} \theta \sin ^{2 k_{m+1}+1} \theta d \theta=\frac{1}{2^{n-1}} \frac{k !\left(k_{1}+1\right)}{(|k|+n) !} .
$$

Putting the pieces together, we have

$$
\alpha_{k}=\sqrt{\pi}\left(k_{1}+1\right)^{1 / 2} \frac{\{2(|k|+n)\} !}{4^{|k|+n}\left[(|k|+n) ! 1^{2}\right.} .
$$


To complete our analysis, we need Stirling's Formula in the form [WW]

$$
m !=m^{m+1 / 2} e^{-m} e^{\theta(m) / 12 m} \sqrt{2 \pi},
$$

where $0<\theta(m)<1$. This gives

$$
\alpha_{k}=\left(k_{1}+1\right)^{1 / 2}(|k|+n)^{-1 / 2} e^{\delta(|k|) /(|k|+n)},
$$

where $|\delta(|k|)| \leqq 1 / 6$. Thus, we have

$$
\alpha_{k}-\beta_{k}=\left(k_{1}+1\right)^{1 / 2}\left\{(|k|+n)^{-1 / 2} e^{\delta(|k|) /(|k|+n)}-(|k|+n+1)^{-1 / 2}\right\}
$$

with $|\delta(|k|)| \leqq 1 / 6$. Using $e^{-x} \geqq 1-x$ for $x \geqq 0$, we see that

$$
\alpha_{k}-\beta_{k} \geqq 0
$$

and, using $e^{x} \leqq 1+3 x$ for $0 \leqq x \leqq 1$, we can check that

$$
\alpha_{k}-\beta_{k} \leqq(|k|+n)^{-1} \text {. }
$$

This allows us to conclude that $D$ is compact.

We also have

Lemma 2. If $p$ is any polynomial in $z_{1}, \ldots, z_{n}, \bar{z}_{1}, \ldots, \bar{z}_{n}$ which is homogeneous of degree $k$, then

$$
T_{|z|^{-k} p}-V \tilde{T}_{p} V^{-1}
$$

is compact.

Proof. The functions $z_{j} /|z|$ are ESV in the sense of [BC2,Theorem 3]. Note that by [BC2, Theorem 11],

$$
T_{f} T_{g}-T_{f g}
$$

is compact for $f, g$ in ESV and ESV is a $*$-algebra under the usual pointwise operations on functions. It follows from [C1, Theorem 1] and Lemma 1 that the desired result holds.

For $g$ in the sup-norm algebra $C\left(S^{2 n-1}\right)$ of continuous complex-valued functions on $S^{2 n-1}$, we define

$$
\hat{g}(z)=g(z /|z|)
$$

on $\mathbf{C}^{n} \backslash\{0\}$. Note that for $p(z)$ a homogeneous polynomial in $z_{1}, \ldots, z_{n}, \bar{z}_{1}, \ldots, \bar{z}_{n}$ of degree $l$,

$$
\hat{p}(z)=|z|^{-l} p(z)
$$

for all $z$ in $\mathbf{C}^{n} \backslash\{0\}$. It is known that $\hat{g}$ is in ESV of [BC2].

We write $\tau\left(\mathbf{B}_{2 n}\right)$ for the $C^{*}$-algebra generated by all $\tilde{T}_{f}$ with $f$ continuous on $\overline{\mathbf{B}}_{2 n}=\mathbf{B}_{2 n} \cup S^{2 n-1}$. This algebra was studied in [C1] and [V].

We will use the definitions of $[\mathrm{BDF}]$ without much discussion. Recall that an exact sequence of $C^{*}$-algebras

$$
0 \rightarrow \mathscr{K} \rightarrow \mathscr{A} \rightarrow C(X) \rightarrow 0
$$

where $\mathscr{K}$ is the full algebra of compact operators and $C(X)$ is the sup-norm algebra of all continuous complex-valued functions on the compact, separable metric space 
$X$, defines an element of $\operatorname{Ext}(X)$. For a Hilbert space $H$, let $B(H)$ denote the collection of all bounded operators on this space. Let $\pi$ denote the quotient map from $B(H)$ to the Calkin algebra $B(H) / K$. It is well-known that $\tau\left(\mathbf{B}_{2 n}\right)$ is an element of $\operatorname{Ext}\left(S^{2 n-1}\right)[\mathrm{C} 1, \mathrm{~V}]$. Indeed this element is represented by the $*$-isomorphism

$$
\tau(f)=\pi\left(\tilde{T}_{f_{e}}\right)
$$

from $C\left(S^{2 n-1}\right)$ into the Calkin algebra $B\left(H^{2}\left(\mathbf{B}_{2 n}\right)\right) / K$, where $f_{e}$ is any continuous extension of $f$ to $\overline{\mathbf{B}}_{2 n}$.

We now have our main technical result.

Representation Theorem 1. For $g$ in $C\left(S^{2 n-1}\right)$,

$$
V^{-1} T_{\hat{g}} V-\tilde{T}_{g_{e}}
$$

is compact for $g_{e}$ any continuous extension of $g$ to $\overline{\mathbf{B}}_{2 n}$.

Proof. This is immediate from Lemma 2 above and [C1,Theorem 1]. We simply choose a sequence of polynomials $\left\{p_{k}\right\}$ so that

$$
\left.p_{k}\right|_{S^{2 n-1}} \rightarrow g
$$

uniformly. It follows that

$$
V^{-1} T_{\hat{p}_{k}} V \rightarrow V^{-1} T_{\hat{g}} V
$$

in norm. By Lemma $2, V^{-1} T_{\hat{g}} V$ is in $\tau\left(\mathbf{B}_{2 n}\right)$. Moreover,

$$
\pi\left(\tilde{T}_{p_{k}}\right) \rightarrow \pi\left(V^{-1} T_{\hat{g}} V\right)
$$

and

$$
\pi\left(\tilde{T}_{p_{k}}\right) \rightarrow \pi\left(\tilde{T}_{g_{e}}\right)
$$

in norm, and the desired result follows.

We now have, for $M_{r}$ the full algebra of $r \times r$ matrices and matrix Toeplitz operators defined in the obvious way:

Corollary 1. For $g$ in $C\left(S^{2 n-1}\right) \otimes M_{r}, T_{\hat{g}}$ is Fredholm if and only if $g$ is invertiblevalued. If $g$ is invertible-valued, then

$$
\operatorname{index}\left(T_{\hat{g}}\right)=(-1)^{n} \text { mapping degree }(g) \text {. }
$$

Proof. Immediate from Theorem 1 above and [V,Theorem 1.5].

For $z=\left(z_{1}, \ldots, z_{n}\right)$ in $\mathbf{C}^{n}$, we write $t_{1}(z)=z_{1}$ and

$$
t_{j}(z)=\left(\begin{array}{cc}
t_{j-1}(z) & -\bar{z}_{j} I \\
z_{j} I & t_{j-1}^{*}(z)
\end{array}\right),
$$

where $I$ is the $2^{j-2} \times 2^{j-2}$ identity matrix and $2 \leqq j \leqq n$. Then the $2^{n-1} \times 2^{n-1}$ matrix function $t_{n}(z)$ is unitary on the unit sphere $S^{2 n-1}$ and generates $K^{1}\left(S^{2 n-1}\right)$ [V]. Moreover, the entries of $t_{n}(z)$ are either 0 or polynomials of degree one in $\left\{z_{j}, \bar{z}_{j}: j=1,2, \ldots, n\right\}$. It follows that

$$
t(z) \equiv|z|^{-1} t_{n}(z)
$$


is a homogeneous function of degree 0 and

$$
\left.\left.t\right|_{S^{2 n-1}} \equiv t_{n}\right|_{S^{2 n-1}}
$$

Corollary 2. The operator $T_{t}$ on $H^{2}\left(\mathbf{C}^{n}\right) \otimes M_{2^{n-1}}$ is Fredholm with

$$
\text { index }\left(T_{t}\right)=(-1)^{n} \text {. }
$$

Proof. By Theorem 5.1 of [V], we have $\tilde{T}_{t_{n}}$ Fredholm with index $\left(\tilde{T}_{t_{n}}\right)=(-1)^{n}$. It follows immediately from Theorem 1 that $T_{t}$ is also Fredholm, with

$$
\operatorname{index}\left(T_{t}\right)=\operatorname{index}\left(\tilde{T}_{t_{n}}\right)=(-1)^{n}
$$

\section{Rieffel Deformations}

In the interest of completeness, we provide a brief discussion of certian aspects of the Rieffel construction which are central to this application.

Suppose that $A$ is a $C^{*}$-algebra on which a vector space $V$ of real dimension $2 n$ acts via a group of automorphisms $\alpha=\left\{\alpha_{x}: x \in V\right\}$. Assume that $V$ is equipped with the usual topology which makes it a topological vector space and that the action of $\alpha$ is strongly continuous. That is, for every $a \in A, x \mapsto \alpha_{x}(a)$ is a continuous map from $V$ to $A$. Suppose that $J$ is a skew-symmetric operator on $V$. Rieffel showed in [R] that given such data $\{A, V, \alpha, J\}$, one can always produce a new $C^{*}$-algebra $A_{J}$ by deforming the original product on a smooth subalgebra of $A$. The $C^{*}$-algebra $A_{J}$ is constructed in the following way.

Let $\mathscr{S}^{A}$ denote the collection of $A$-valued functions $f$ on $V$ which, together with its partial derivations of all orders, rapidly decrease to 0 at infinity. For $f \in \mathscr{S}^{A}$, define

$$
\|f\|_{2}=\left\|\int_{V} f(x)^{*} f(x) d x\right\|^{1 / 2} .
$$

Let $A^{\infty}$ denote the collection of elements $a \in A$ such that the $A$-valued function $x \mapsto \alpha_{x}(a)$ is $C^{\infty}$ on $V$. Each $a \in A^{\infty}$ gives rise to an operator

$$
\left(L_{a} f\right)(x)=(2 \pi)^{-(\operatorname{dim} V) / 2} \int_{V} \int_{V} \alpha_{x+J u}(a) f(x+v) e^{i u} \cdot{ }^{v} d v d u
$$

on $\mathscr{S}^{A}$. It is easy to check that for any $a, b \in A^{\infty}$, we have

$$
L_{a} L_{b}=L_{a \times J b}
$$

where

$$
a \times_{J} b=(2 \pi)^{-(\operatorname{dim} V) / 2} \int_{V} \int_{V} \alpha_{J u}(a) \alpha_{v}(b) e^{i u \cdot v} d v d u .
$$

The above is known as an oscillatory integral and its convergence for $a, b \in A^{\infty}$ was shown in $[R]$. Rieffel also showed in $[R]$ that

$$
\|a\|_{J}=\left\|L_{a}\right\|=\sup \left\{\left\|L_{a} f\right\|_{2}: f \in \mathscr{S}^{A},\|f\|_{2}=1\right\}
$$

is a $C^{*}$-norm on $A^{\infty}$. Therefore with the product $\times_{J}$ and the norm $\|\cdot\|_{J}, A^{\infty}$ becomes a pre- $C^{*}$-algebra. The $C^{*}$-algebra $A_{J}$, i.e., the Rieffel deformation of $A$, is defined to be the completion of $A^{\infty}$ with respect to the norm $\|\cdot\|_{J}$. We may, 
of course, also regard $A_{J}$ as the completion of $\left\{L_{a}: a \in A^{\infty}\right\}$ with respect to the operator norm on $\mathscr{S}^{A}$.

Given $\{A, V, \alpha, J\}$ a Poisson bracket $\{\cdot, \cdot\}$ can be constructed on $A^{\infty}$ as follows. Fix a basis $x_{1}, \ldots, x_{d}$ for $V$ so that $J$ is represented by a skew-symmetric matrix $\left(J_{j k}\right)$ with respect to this basis. Let $X_{1}, \ldots, X_{d}$ be the basis dual to $x_{1}, \ldots, x_{d}$ in the Lie algebra $L$ of $V$. Accordingly, we have the infinitesimal generators $\alpha_{X_{1}}, \ldots, \alpha_{X_{d}}$ of the automorphism group $\alpha$. That is, for any $a \in A^{\infty}$,

$$
\alpha_{X_{j}}(a)=\lim _{t \rightarrow 0} \frac{1}{t}\left(\alpha_{t x_{j}}(a)-a\right)
$$

$1 \leqq j \leqq d$. Then

$$
\{a, b\}=\sum_{1 \leqq j, k \leqq d} J_{j k} \alpha_{X}(a) \alpha_{X_{k}}(b), \quad a, b \in A^{\infty},
$$

defines a Poisson bracket on $A^{\infty}$.

For $\hbar$ a real parameter, $\hbar J$ is also a skew-symmetric operator on $V$. Therefore we also have the deformed product $\times_{\hbar J}$ and the norm $\|\cdot\|_{\hbar_{J}}$ on $A^{\infty}$. It was shown in [R] that the family $\left\{\left(A^{\infty}, \times_{\hbar J},\|\cdot\|_{\hbar J}\right): 0<|\hbar| \leqq 1\right\}$ forms a strict deformation quantization of $A^{\infty}$ in the direction of $\{\cdot, \cdot\}$ in the following sense:

(1) For every $a \in A^{\infty}$, the map $\hbar \mapsto\|a\|_{\hbar J}$ is continuous.

(2) For every pair, $a, b \in A^{\infty}$,

$$
\lim _{\hbar \rightarrow 0}\left\|\frac{1}{i \hbar}\left(a \times_{\hbar J} b-a b\right)-\{a, b\}\right\|_{\hbar J}=0 .
$$

It turns out that the deformed algebra $A_{J}$ can be quite interesting even when $A$ itself is rather ordinary. For example, if one takes $A=A\left(\mathbf{R}^{2}\right)$ to be the collection of continuous functions $f$ on $\mathbf{C}=\mathbf{R}^{2}$ such that the limit

$$
\lim _{R \rightarrow+\infty} f(R z)
$$

exists uniformly on the circle $\{z \in \mathbf{C}:|z|=1\}, \alpha$ to be the natural translation of $\mathbf{R}^{2}$, and $J(x, y)=(y,-x)$, then, as was shown in $[\mathrm{R}], A_{J}=A_{J}\left(\mathbf{R}^{2}\right)$ is isomorphic to the Toeplitz algebra on the Bergmann space of the unit disc. The main purpose of this paper is to show that the analogous result holds for $\mathbf{C}^{n}=\mathbf{R}^{2 n}$.

We now make precise the connection to Rieffel's construction of $A_{J}\left(\mathbf{R}^{2 n}\right)$. To ease notation, we will simply write $A_{J}$ instead of $A_{J}\left(\mathbf{R}^{2 n}\right)$ for the rest of the section.

From now on we let $A$ be the collection of continuous functions $f$ on $\mathbf{R}^{2 n}$ which have the property that the radial limit

$$
f_{\text {radial }}(w)=\lim _{R \rightarrow+\infty} f(R w)
$$

exists uniformly on the sphere $S^{2 n-1}$. The vector space $V=\mathbf{R}^{2 n}$ acts on $A$ by the natural translation. Let $J$ be the standard simplectic operator on $\mathbf{R}^{2 n}$. That is,

$$
J\left(x_{1}, y_{1}, \ldots, x_{n}, y_{n}\right)=\left(y_{1},-x_{1}, \ldots, y_{n},-x_{n}\right) .
$$

Let $A^{\infty}$ denote the collection of $f \in A$ such that $z \mapsto f(.+z)$ is a $C^{\infty}$-map from $\mathbf{R}^{2 n}$ into $A$ Accordinolv $\mathscr{P}^{A}$ consists of $A$-valued smonth functions on $\mathbf{R}^{2 n}$ which. 
together with all their derivatives, are rapidly decreasing. By Rieffel's construction, each $f \in A^{\infty}$ gives rise to an operator $L_{f}$ on $\mathscr{S}^{A}$ :

$$
\left(L_{f} g\right)(x, z)=(2 \pi)^{-n} \int_{\mathbf{R}^{2 n}} \int_{\mathbf{R}}^{2 n} f(z+x+J u) g(x+v, z) e^{i u} \cdot v d u d v
$$

(For each $x \in \mathbf{R}^{2 n}, g(x, \cdot)$ denotes the value of $g$ at $x$, which is an element in $A$.) The $C^{*}$-algebra $A_{J}$ is defined to be the completion of $\left\{L_{f}: f \in A^{\infty}\right\}$ with respect to the norm $\|f\|_{J}=\left\|L_{f}\right\|=\sup \left\{\left\|L_{f} g\right\|_{\mathscr{S} A}: g \in \mathscr{S}^{A},\|g\|_{\mathscr{S}^{A}}=1\right\}$. Let $\mathscr{S}\left(\mathbf{R}^{2 n}\right)$ denote the collection of smooth, rapidly decreasing functions on $\mathbf{R}^{2 n}$. For each $f \in A^{\infty}$ and each $z \in \mathbf{R}^{2 n}$, we can define a Weyl operator

$$
\left(W_{f}^{z} \eta\right)(x)=(2 \pi)^{-n} \int_{\mathbf{R}^{2 n} \mathbf{R}^{2 n}} f(z+x+J u) \eta(x+v) e^{i u \cdot v} d u d v
$$

on $\mathscr{S}\left(\mathbf{R}^{2 n}\right) . W_{f}^{x}$ extends to a bounded operator on $L^{2}\left(\mathbf{R}^{2 n}\right)$. Indeed because $\mathscr{S}\left(\mathbf{R}^{2 n}\right)$ is actually a subset of $\mathscr{S}^{A}$, by the definition of the norm on $\mathscr{S}^{A}$ and the definition of the $L^{2}$-norm, it is obvious that $\left\|W_{f}^{z}\right\| \leqq\left\|L_{f}\right\|$. The norm $\left\|W_{f}^{z}\right\|$ is independent of $z$. This can be seen in the following way.

Define the unitary operator $\left(U_{z} \xi\right)(x)=\xi(x+z)$ on $L^{2}\left(\mathbf{R}^{2 n}\right)$. Then it is straightforward to verify that

$$
U_{z} W_{f}^{0} U_{-z}=W_{f}^{z} .
$$

This equality also implies that

$$
\left\|W_{f}^{z}\right\|=\left\|L_{f}\right\|
$$

Indeed by the definition of the norm on $\mathscr{S}^{A}$, we have

$$
\begin{aligned}
\left\|L_{f} g\right\|_{\mathscr{S}^{A}}^{2} & =\sup _{z \in \mathbf{R}^{2 n} \mathbf{R}^{2 n}}\left|(2 \pi)^{-n} \int_{\mathbf{R}^{2 n} \mathbf{R}^{2 n}} f(z+x+J u) g(x+v, z) e^{i u} \cdot v d u d v\right|^{2} d x \\
& =\sup _{z \in \mathbf{R}^{2 n}}\left\|W_{f}^{z} g_{z}\right\|_{2}^{2} \leqq\left\|W_{f}^{0}\right\| \sup _{z \in \mathbf{R}^{2 n}}\left\|g_{z}\right\|_{2}^{2}=\left\|W_{f}^{0}\right\|\|g\|_{\mathscr{\mathscr { S } _ { A }}}^{2},
\end{aligned}
$$

where $g_{z}$ denotes the element $g_{z}(x)=g(x, z)$ in $\mathscr{S}\left(\mathbf{R}^{2 n}\right)$.

It is straightforward to verify that for $f_{1}, f_{2} \in A^{\infty}$,

$$
W_{f_{1}}^{z} W_{f_{2}}^{z}=W_{f_{1} \times f_{2}}^{z},
$$

where $f_{1} \times_{J} f_{2}$ is the deformation product described earlier. Hence for every $z \in$ $\mathbf{R}^{2 n}$, the map

$$
\pi_{z}: L_{f} \mapsto W_{f}^{z}
$$

extends to a $C^{*}$-algebra isomorphism from $A_{J}$ onto a subalgebra of $B\left(L^{2}\left(\mathbf{R}^{2 n}\right)\right)$. We will next show that each $\pi_{z}\left(A_{J}\right)$ is isomorphic to a $C^{*}$-algebra of pseudo-differential operators of order zero on $L^{2}\left(\mathbf{R}^{n}\right)$. Obviously it suffices to do this for the case $z=0$.

For each $j=1, \ldots, n$, define

$$
\begin{aligned}
\left(M_{j}^{1} f\right)\left(s_{1}, t_{1}, \ldots, s_{n}, t_{n}\right) & =s_{j} f\left(s_{1}, t_{1}, \ldots, s_{n}, t_{n}\right), \\
\left(M_{j}^{2} f\right)\left(s_{1}, t_{1}, \ldots, s_{n}, t_{n}\right) & =t_{j} f\left(s_{1}, t_{1}, \ldots, s_{n}, t_{n}\right), \\
\left(\partial_{j}^{1} f\right)\left(s_{1}, t_{1}, \ldots, s_{n}, t_{n}\right) & =-i \frac{\partial}{\partial s_{j}} f\left(s_{1}, t_{1}, \ldots, s_{n}, t_{n}\right),
\end{aligned}
$$


and

$$
\left(\partial_{j}^{2} f\right)\left(s_{1}, t_{1}, \ldots, s_{n}, t_{n}\right)=-i \frac{\partial}{\partial t_{j}} f\left(s_{1}, t_{1}, \ldots, s_{n}, t_{n}\right) .
$$

Furthermore, we define

$$
D_{j}^{1}=M_{j}^{1}-\partial_{j}^{2}
$$

and

$$
D_{j}^{2}=M_{j}^{2}+\partial_{j}^{1},
$$

$j=1, \ldots, n$. We have the commutation relations

$$
D_{j}^{1} D_{j}^{2}-D_{j}^{2} D_{j}^{1}=2 i
$$

for all $j$ and

$$
D_{j}^{p} D_{k}^{q}-D_{k}^{q} D_{j}^{p}=0
$$

for all $j \neq k$ and $p, q=1,2$. Let $e_{j}^{1}$ (resp. $e_{j}^{2}$ ) be the vector in $\mathbf{R}^{2 n}$ whose $(2 j-1)^{\text {st }}$ (resp. $2 j^{\text {th }}$ ) coordinate is 1 and whose other coordinates are 0 . Then it is straightforward to verify that

$$
\left(\exp \left(i s_{j} D_{j}^{1}\right) \eta\right)(x)=\frac{1}{2 \pi} \iint_{\mathbf{R}} \exp \left(i s_{j}\left(x_{2 j-1}+u_{2 j}\right)\right) \eta\left(x+v_{2 j} e_{j}^{2}\right) \exp \left(i u_{2 j} v_{2 j}\right) d u_{2 j} d v_{2 j}
$$

and

$$
\begin{aligned}
& \left(\exp \left(i t_{j} D_{j}^{2}\right) \eta\right)(x) \\
& \quad=\frac{1}{2 \pi} \iint_{\mathbf{R}} \exp \left(i t_{j}\left(x_{2 j}-u_{2 j-1}\right)\right) \eta\left(x+v_{2 j-1} e_{j}^{1}\right) \exp \left(i u_{2 j-1} v_{2 j-1}\right) d u_{2 j-1} d v_{2 j-1}
\end{aligned}
$$

for every $\eta \in \mathscr{S}\left(\mathbf{R}^{2 n}\right)$. By the commutation relation (1), we have

$$
\begin{aligned}
\left(\exp \left(i s_{j} D_{j}^{1}\right) \exp \left(i t_{j} D_{j}^{2}\right) \eta\right)(x) \\
=\exp \left(i s_{j} D_{j}^{1}\right) \exp \left(i t_{j} M_{j}^{2}\right) \frac{1}{2 \pi} \int_{\mathbf{R}} \int_{\mathbf{R}} \exp \left(-i t_{j} u_{2 j-1}\right) \eta\left(x+v_{2 j-1} e_{j}^{1}\right) \\
\quad \times \exp \left(i u_{2 j-1} v_{2 j-1}\right) d u_{2 j-1} d v_{2 j-1} \\
=\exp \left(-i s_{j} t_{j}\right) \exp \left(i t_{j} M_{j}^{2}\right) \exp \left(i s_{j} D_{j}^{1}\right) \frac{1}{2 \pi} \iint_{\mathbf{R}} \exp \left(-i t_{j} u_{2 j-1}\right) \eta\left(x+v_{2 j-1} e_{j}^{1}\right) \\
\quad \times \exp \left(i u_{2 j-1} v_{2 j-1}\right) d u_{2 j-1} d v_{2 j-1} \\
=\exp \left(-i s_{j} t_{j}\right) \exp \left(i t_{j} x_{2 j}\right)(2 \pi)^{-2} \iiint_{\mathbf{R}} \int_{\mathbf{R}} \exp \left(i s_{j}\left(x_{2 j-1}+u_{2 j}\right)\right) \exp \left(-i t_{j} u_{2 j-1}\right) \\
\quad \times \eta\left(x+v_{2 j-1} e_{j}^{1}+v_{2 j} e_{J}^{2}\right) \exp \left(i\left[u_{2 j-1} v_{2 j-1}+u_{2 j} v_{2 j}\right]\right) d u_{2 j-1} d v_{2 j-1} d u_{2 j} d v_{2 j} \\
=\exp \left(-i s_{j} t_{j}\right)(2 \pi)^{-2} \iint_{\mathbf{R}} \int_{\mathbf{R}} \int_{\mathbf{R}} \exp \left(i\left(s_{j} e_{j}^{1}+t_{j} e_{j}^{2}\right) \cdot\left(x+J\left(u_{2 j-1} e_{j}^{1}+u_{2 j} e_{j}^{2}\right)\right)\right) \\
\quad \times \eta\left(x+v_{2 j-1} e_{j}^{1}+v_{2 j} e_{j}^{2}\right) \exp \left(i\left(u_{2 j-1} e_{j}^{1}+u_{2 j} e_{j}^{2}\right)\right. \\
\left.\quad \cdot\left(v_{2 j-1} e_{j}^{1}+v_{2 j} e_{j}^{2}\right)\right) d u_{2 j-1} d v_{2 j-1} d u_{2 j} d v_{2 j}
\end{aligned}
$$


Suppose that $s=\left(s_{1}, \ldots, s_{n}\right)$ and $t=\left(t_{1}, \ldots, t_{n}\right)$. By the commutation relation (2) and the above identity, we have

$$
\begin{aligned}
& \left(\prod_{j=1}^{n} \exp \left(i s_{j} D_{j}^{1}\right) \exp \left(i t_{j} D_{j}^{2}\right) \eta\right)(x) \\
& \quad=e^{-i s \cdot{ }^{t}}(2 \pi)^{-n} \int_{\mathbf{R}^{2 n} \mathbf{R}^{2 n}} e^{l(A s+B t) \cdot(x+J u)} \eta(x+v) e^{i u} \cdot v d u d v .
\end{aligned}
$$

Here, $A, B: \mathbf{R}^{n} \rightarrow \mathbf{R}^{2 n}$ are the linear transformations defined by the formulas

$$
A\left(s_{1}, \ldots, s_{n}\right)=\left(s_{1}, 0, \ldots, s_{n}, 0\right)
$$

and

$$
B\left(t_{1}, \ldots, t_{n}\right)=\left(0, t_{1}, \ldots, 0, t_{n}\right) .
$$

If $b \in \mathscr{S}\left(\mathbf{R}^{n} \times \mathbf{R}^{n}\right)\left(\cong \mathscr{S}\left(\mathbf{R}^{2 n}\right)\right)$, then

$$
\begin{aligned}
& \left(\left[\int_{\mathbf{R}^{n}} \int_{\mathbf{R}^{n}} b(s, t) \prod_{j=1}^{n} \exp \left(i s_{j} D_{j}^{1}\right) \exp \left(i t_{j} D_{j}^{2}\right) d s d t\right] \eta\right)(x) \\
& =(2 \pi)^{-n} \int_{\mathbf{R}^{2 n} \mathbf{R}^{2 n}}\left[\int_{\mathbf{R}^{n} \mathbf{R}^{n}} b(s, t) e^{i((A s+B t) \cdot(x+J u)-s \cdot t)} d s d t\right] \eta(x+v) e^{i u} \cdot v d u d v .
\end{aligned}
$$

In other words, if we define

$$
(\Phi b)(x)=\iint_{\mathbf{R}^{n} \mathbf{R}^{n}} b(s, t) e^{l((A s+B t) \cdot x-s \cdot t)} d s d t,
$$

then

$$
\int_{\mathbf{R}^{n} \mathbf{R}^{n}} b(s, t) \prod_{j=1}^{n} \exp \left(i s_{j} D_{j}^{1}\right) \exp \left(i t_{j} D_{j}^{2}\right) d s d t=W_{\Phi b}^{0} .
$$

Suppose now that $a, b \in \mathscr{S}\left(\mathbf{R}^{n} \times \mathbf{R}^{n}\right)$. Then, by (1) and (2), we have

$$
\begin{aligned}
& W_{(\Phi a) \times_{J}(\Phi b)}^{0}=W_{\Phi a}^{0} W_{\Phi b}^{0}
\end{aligned}
$$

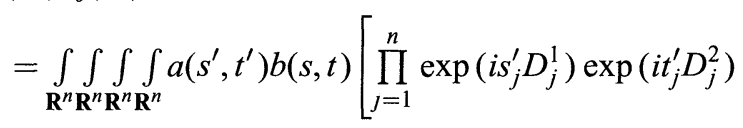

$$
\begin{aligned}
& \left.\times \exp \left(i s_{j} D_{j}^{1}\right) \exp \left(i t_{j} D_{j}^{2}\right)\right] d s^{\prime} d t^{\prime} d s d t \\
& =\iint_{\mathbf{R}^{n} \mathbf{R}^{n} \mathbf{R}^{n} \mathbf{R}^{n}} a\left(s^{\prime}, t^{\prime}\right) b(s, t)\left[\prod_{j=1}^{n} \exp \left(i s_{j}^{\prime} D_{j}^{1}\right) \exp \left(i s_{j} D_{j}^{1}\right) \exp \left(i t_{j}^{\prime} D_{j}^{2}\right) \exp \left(2 i s_{j} t_{j}^{\prime}\right)\right. \\
& \left.\times \exp \left(i t_{j} D_{j}^{2}\right)\right] d s^{\prime} d t^{\prime} d s d t \\
& =\iint_{\mathbf{R}^{n} \mathbf{R}^{n} \mathbf{R}^{n} \mathbf{R}^{n}} a\left(s^{\prime}, t^{\prime}\right) b(s, t) e^{2 i s} \cdot t\left[\prod_{j=1}^{n} \exp \left(i\left(s_{j}^{\prime}+s_{j}\right) D_{j}^{1}\right)\right. \\
& \left.\exp \left(i\left(t_{j}^{\prime}+t_{j}\right) D_{j}^{2}\right)\right] d s^{\prime} d t^{\prime} d s d t
\end{aligned}
$$




$$
\begin{aligned}
= & \int_{\mathbf{R}^{n} \mathbf{R}^{n}} \int\left[\int_{\mathbf{R}^{n} \mathbf{R}^{n}} a\left(s^{\prime}, t^{\prime}\right) b\left(s-s^{\prime}, t-t^{\prime}\right) e^{2 l\left(s-s^{\prime}\right) \cdot t^{\prime}} d s^{\prime} d t^{\prime}\right] \\
& \times\left[\prod_{j=1}^{n} \exp \left(i s_{j} D_{J}^{1}\right) \exp \left(i t_{j} D_{j}^{2}\right)\right] d s d t \\
= & \int_{\mathbf{R}^{n}} \int_{\mathbf{R}^{n}}(a * b)(s, t)\left[\prod_{j=1}^{n} \exp \left(i s_{j} D_{j}^{1}\right) \exp \left(i t_{j} D_{j}^{2}\right)\right] d s d t,
\end{aligned}
$$

where

$$
(a * b)(s, t)=\int_{\mathbf{R}^{n} \mathbf{R}^{n}} a\left(s^{\prime}, t^{\prime}\right) b\left(s-s^{\prime}, t-t^{\prime}\right) e^{2 i\left(s-s^{\prime}\right) \cdot t^{\prime}} d s^{\prime} d t^{\prime}
$$

Hence

$$
(\Phi a) \times_{J}(\Phi b)=\Phi(a * b) .
$$

We will now establish the relations between $W_{f}^{x}$ and the Weyl operators on $L^{2}\left(\mathbf{R}^{n}\right)$. Following [R], we define a Weyl operator on $L^{2}\left(\mathbf{R}^{n}\right)$ with symbol function $\alpha \in \mathscr{S}\left(\mathbf{R}^{n} \times \mathbf{R}^{n}\right)$ by the formula

$$
\left(\Psi_{\alpha} \xi\right)(x)=(2 \pi)^{-n} \int_{\mathbf{R}^{n} \int^{n}} \alpha(x+y, v) e^{l(x-y) \cdot v} \xi(y) d y d v
$$

for $\xi \in \mathscr{S}\left(\mathbf{R}^{n}\right)$. Also recall that for $\alpha, \beta \in \mathscr{S}\left(\mathbf{R}^{n} \times \mathbf{R}^{n}\right)$, the Weyl symbol calculus $\alpha \times_{W} \beta$ is defined by the relation

$$
\Psi_{\alpha \times_{W} \beta}=\Psi_{\alpha} \Psi_{\beta}
$$

This relation is still valid if one of $\alpha, \beta$ is only a function in $A^{\infty}$.

It follows from (1) and (2) that there is a unitary operator $Y: L^{2}\left(\mathbf{R}^{2 n}\right) \rightarrow$ $L^{2}\left(\mathbf{R}^{2 n}\right)$ such that

$$
Y D_{j}^{1} Y^{*}=2 M_{j}^{1} \quad \text { and } \quad Y D_{j}^{2} Y^{*}=\partial_{j}^{1} .
$$

(Because of (2), one only needs to prove this in the case $n=1$. But in this case such a $Y$ can be constructed explicitly. See, for example, $[\mathrm{X}]$.) We can identify $M_{j}^{1}$ with $m_{j} \otimes 1$ and $\partial_{j}^{1}$ with $d_{J} \otimes 1$, where $m_{j}$ and $d_{J}$ are the operators

$$
\left(m_{j} f\right)\left(x_{1}, \ldots, x_{n}\right)=x_{j} f\left(x_{1}, \ldots, x_{n}\right)
$$

and

$$
\left(d_{j} f\right)\left(x_{1}, \ldots, x_{n}\right)=-i \frac{\partial}{\partial x_{j}} f\left(x_{1}, \ldots, x_{n}\right)
$$

on $L^{2}\left(\mathbf{R}^{n}\right)$. Thus, we have shown that for any $b \in \mathscr{S}\left(\mathbf{R}^{n} \times \mathbf{R}^{n}\right)$,

$$
Y W_{\Phi b}^{0} Y^{*}=\left[\int_{\mathbf{R}^{n} \mathbf{R}^{n}} b(s, t) \prod_{j=1}^{n} \exp \left(2 i s_{j} m_{j}\right) \exp \left(i t_{j} d_{j}\right) d s d t\right] \otimes 1 .
$$

Since

$$
b(s, t)=\exp (i s \cdot t)(2 \pi)^{-n} \int_{\mathbf{R}^{n} \int^{n}}(\Phi b)(A u+B v) e^{-l[s \cdot u+t \cdot v]} d u d v
$$


for any $\xi \in \mathscr{S}\left(\mathbf{R}^{n}\right)$, we have

$$
\begin{aligned}
& \left(\left[\int_{\mathbf{R}^{n} \mathbf{R}^{n}} b(s, t) \prod_{j=1}^{n} \exp \left(2 i s_{j} m_{j}\right) \exp \left(i t_{j} d_{j}\right) d s d t\right] \xi\right)(x) \\
& =\iint_{\mathbf{R}^{n} \mathbf{R}^{n}} b(s, t) e^{2 i s \cdot x} \xi(x+t) d s d t \\
& =(2 \pi)^{-n} \int_{\mathbf{R}^{n} \mathbf{R}^{n}} e^{2 i s \cdot x}\left[e^{i s \cdot t}\right. \\
& \left.\times \int_{\mathbf{R}^{n} \mathbf{R}^{n}}(\Phi b)(A u+B v) e^{-i[s \cdot u+t \cdot v]} d u d v\right] \xi(x+t) d s d t \\
& =(2 \pi)^{-n} \int_{\mathbf{R}^{n}} \int_{\mathbf{R}^{n}} \int_{\mathbf{R}^{n} \mathbf{R}^{n}}(\Phi b)(A u+B v) e^{-i[s \cdot(u-x-t)+(t-x) \cdot v]} \xi(t) d u d v d s d t \\
& =(2 \pi)^{-n} \iint_{\mathbf{R}^{n} \mathbf{R}^{n}{ }_{\mathbf{R}^{n}}}(\Phi b)(A(u+t+x)+B v) e^{-i[s \cdot u+(t-x) \cdot v]} \xi(t) d u d v d s d t
\end{aligned}
$$

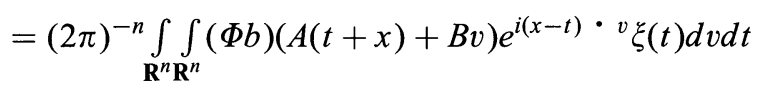

$$
\begin{aligned}
& =(2 \pi)^{-n} \int_{\mathbf{R}^{n} \mathbf{R}^{n}}(T \Phi b)(t+x, v) e^{i(x-t)} \cdot v \xi(t) d v d t .
\end{aligned}
$$

Here, we use the notation

$$
(T F)(u, v)=F(A u+B v), u, v \in \mathbf{R}^{n},
$$

for functions $F$ defined on $\mathbf{R}^{2 n}\left({ }^{1}\right)$. Hence it follows from this calculation and (5) that

$$
Y W_{\Phi b}^{0} Y^{*}=\Psi_{T \Phi b} \otimes 1
$$

It follows from (4) and the commutation relations (1) and (2) that, for any $a, b \in$ $\mathscr{S}\left(\mathbf{R}^{n} \times \mathbf{R}^{n}\right)$,

$$
\begin{aligned}
& \Psi_{(T \Phi a) \times{ }_{W}(T \Phi b)}=\Psi_{T \Phi a} \Psi_{T \Phi b} \\
& =\iint_{\mathbf{R}^{n} \mathbf{R}^{n} \mathbf{R}^{n} \mathbf{R}^{n}} a\left(s^{\prime}, t^{\prime}\right) b(s, t) \\
& \quad \times\left[\prod_{j=1}^{n} \exp \left(2 i s_{j}^{\prime} m_{j}\right) \exp \left(i t_{j}^{\prime} d_{j}\right) \exp \left(2 i s_{j} m_{j}\right) \exp \left(i t_{j} d_{j}\right)\right] d s^{\prime} d t^{\prime} d s d t \\
& =\int_{\mathbf{R}^{n} \mathbf{R}^{n}}(a * b)(s, t)\left[\prod_{j=1}^{n} \exp \left(2 i s_{j} m_{j}\right) \exp \left(i t_{j} d_{j}\right)\right] d s d t \\
& =\Psi_{T \Phi(a * b)}=\Psi_{T\left[(\Phi a) \times_{J}(\Phi b)\right] .}
\end{aligned}
$$

That is,

$$
(T \Phi a) \times_{W}(T \Phi b)=T\left[(\Phi a) \times_{J}(\Phi b)\right] .
$$

\footnotetext{
${ }^{1}$ It may seem that the introduction of $T$ does nothing but create inconvenience. After all, why don't we simply identify $F(u, v)$ and $F(A u+B v)$ ? What is implicit in such an identification of functions, however, is an identification of $\mathbf{R}^{n} \times \mathbf{R}^{n}$ with $\mathbf{R}^{2 n}$ through a rearrangement of coordinates. There are $(2 n)$ ! ways to do so. The presence of $T$ serves as a reminder of our particular rearrangement of coordinates which was actually dictated by the choice of the operator $J$.
} 
If $f \in A^{\infty}$ (technically, elements of $A^{\infty}$ are functions on $\mathbf{R}^{2 n}$, not functions of the form $\left.F(u, v), u, v \in \mathbf{R}^{n}\right)$, then for any $b \in \mathscr{S}\left(\mathbf{R}^{n} \times \mathbf{R}^{n}\right)$, we also have

$$
(T f) \times_{W}(T \Phi b)=T\left[f \times_{J}(\Phi b)\right]
$$

and

$$
\Psi_{(T f) \times_{W}(T \Phi b)}=\Psi_{T f} \Psi_{T \Phi b} .
$$

Hence it follows from (6) that

$$
\begin{aligned}
Y W_{f}^{0} W_{\Phi b}^{0} Y^{*} & =Y W_{f \times_{J}(\Phi b)}^{0} Y^{*}=\Psi_{T\left[f \times_{J}(\Phi b)\right]} \otimes 1 \\
& =\Psi_{(T f) \times_{W}(T \Phi b)} \otimes 1=\left[\Psi_{T f} \Psi_{T \Phi b}\right] \otimes 1
\end{aligned}
$$

for any $f \in A^{\infty}$ and $b \in \mathscr{S}\left(\mathbf{R}^{n} \times \mathbf{R}^{n}\right)$. By choosing a sequence $\left\{b_{n}\right\} \subset \mathscr{S}\left(\mathbf{R}^{n} \times \mathbf{R}^{n}\right)$ such that $\left\{\Psi_{T \Phi b_{n}}\right\}$ converges to the identity operator strongly, we may conclude that

$$
Y W_{f}^{0} Y^{*}=\Psi_{T f} \otimes 1
$$

It follows from Rieffel's construction that $\left\{L_{f}: f \in A^{\infty}\right\}$ is dense in $A_{J}$. Hence the map

$$
\pi_{J}: L_{f} \mapsto \Psi_{T f}, f \in A^{\infty},
$$

extends to a $C^{*}$-algebra isomorphism from $A_{J}$ into $B\left(L^{2}\left(\mathbf{R}^{n}\right)\right)$.

For $f$ a bounded function (or matrix of such functions) on $\mathbf{C}^{n}$, we recall that the Bargmann isometry [B; F, p. 40; BC3]

$$
B: L^{2}\left(\mathbf{R}^{n}, d v\right) \rightarrow H^{2}\left(C^{n}, d \mu\right)
$$

has the property that

$$
B^{-1} T_{f} B=W_{\beta}
$$

where

$$
\beta(\xi, x)=\tilde{f}(x-i \xi)=\frac{1}{\pi^{n}} \int_{\mathbf{C}^{n}} f(w) e^{-|w-(x-i \xi)|^{2}} d v(w), \quad x, \quad \xi \in \mathbf{R}^{n},
$$

is defined to be the solution of the heat equation at time $t=1 / 4$ with initial-value $f$, and $W_{\beta}$ is the Weyl operator on $L^{2}\left(\mathbf{R}^{n}, d v\right)$ given by

$$
\left(W_{\beta} g\right)(x)=(2 \pi)^{-n} \int_{\mathbf{R}^{n} \mathbf{R}^{n}} \beta\left(\xi, \frac{x+y}{2}\right) e^{i(x-y) \cdot \xi} g(y) d y d \xi
$$

[F, p. 141; BC3].

The definition of the Weyl operator $W_{\beta}$ with the symbol function $\beta$ is slightly different from the definition of $\Psi_{\beta}$. However the two sets of pseudo-differential operators $\left\{\Psi_{T f}: f \in A^{\infty}\right\}$ and $\left\{W_{T f}: f \in A^{\infty}\right\}$ are identical and, therefore, generate the same $C^{*}$-algebra. This fact can be seen from a transformation on $A$. For any $\lambda>$ 0 , define the linear operator $S_{\lambda}$ on $\mathbf{R}^{2 n}$ by the formula $S_{\lambda}\left(x_{1}, \ldots, x_{n}, x_{n+1}, \ldots, x_{2 n}\right)=$ $\left(x_{n+1}, \ldots, x_{2 n}, \lambda x_{1}, \ldots, \lambda x_{n}\right)$. Also define $\left(S_{\lambda *} f\right)(x)=f\left(S_{\lambda} x\right)$ for $f \in A$. If we denote $\left(\alpha_{z}(f)\right)(x)=f(x+z)$, then we have $\alpha_{z}\left(S_{\lambda *} f\right)=S_{\lambda *} \alpha_{S_{\lambda} z}(f)$. Hence if $z \mapsto \alpha_{z}(f)$ is an $A$-valued $C^{\infty}$-function, then so are $z \mapsto \alpha_{S_{\lambda} z}(f)$ and $z \mapsto S_{\lambda *} \alpha_{S_{\lambda} z}(f)$. In other words, the operator $S_{\lambda *}$ maps $A^{\infty}$ to itself. For any $f \in A^{\infty}$, we have

$$
\Psi_{T S_{1 / 2 *} f}=W_{T f} \quad \text { and } \quad W_{T S_{2 *} f}=\Psi_{T f} .
$$


Let $I$ denote the ideal $C_{0}\left(\mathbf{R}^{2 n}\right)$ in $A$. We have an exact sequence

$$
0 \rightarrow I \rightarrow A \rightarrow C\left(S^{2 n-1}\right) \rightarrow 0
$$

It is clear that $I$ is invariant under the action of $\mathbf{R}^{2 n}$ and that the induced action of $\mathbf{R}^{2 n}$ on the quotient algebra $C\left(S^{2 n-1}\right)$ is trivial. Hence by Theorem 7.7 of $[\mathrm{R}]$, we have an induced exact sequence

$$
0 \rightarrow I_{J} \rightarrow A_{J} \rightarrow C\left(S^{2 n-1}\right) \rightarrow 0
$$

If $\pi$ denotes the quotient map from $A_{J}$ onto $C\left(S^{2 n-1}\right)$, then, by Rieffel's construction,

$$
\pi\left(L_{f}\right)=f_{\text {radial }}
$$

for every $f \in A^{\infty}$. We have shown that $\pi_{J}$ is an isomorphism from $A_{J}$ onto the $C^{*}$ algebra generated by $\left\{\Psi_{T f}: f \in A^{\infty}\right\}$. Because $\pi_{J}\left(L_{f}+I_{J}\right)=\Psi_{T f}+\pi_{J}\left(I_{J}\right)$, we see that the image of $\Psi_{T f}$ under the quotient map $\pi_{J}\left(A_{J}\right) \rightarrow \pi_{J}\left(A_{J}\right) / \pi_{J}\left(I_{J}\right)$ is also $f_{\text {radial }}$ for every $f \in A^{\infty}$.

The ideal $\pi_{J}\left(I_{J}\right)$, which is generated by $\left\{\Psi_{T f}: f \in I^{\infty}\right\}$, is the collection of compact operators on $L^{2}\left(\mathbf{R}^{n}\right)$. In fact, if $\alpha \in \mathscr{S}\left(\mathbf{R}^{n} \times \mathbf{R}^{n}\right)$, then obviously $\Psi_{\alpha}$ is a compact operator. From this it is easy to see that $\pi_{J}\left(I_{J}\right)$ at least contains all the compact operators on $L^{2}\left(\mathbf{R}^{n}\right)$. Now, because $I_{J}$ is the completion of $I^{\infty}$ with respect to the $J$-norm, to establish that every operator in $\pi_{J}\left(I_{J}\right)$ is compact, it suffices to verify that $\left\{L_{f}: f \in \mathscr{S}\left(\mathbf{R}^{2 n}\right)\right\}$ is dense in $\left\{L_{f}: f \in I^{\infty}\right\}$. For this purpose, we fix a $C^{\infty}$-function $0 \leqq \eta \leqq 1$ on $[0, \infty)$ such that $\eta=1$ on $[0,1]$ and such that $\eta=0$ on $[2, \infty)$. For each $k \in \mathbf{Z}_{+}$, define $\eta_{k}(t)=1$ if $|t| \leqq k$ and $\eta_{k}(t)=\eta(|t|-k)$ if $|t|>k$. Let

$$
\xi_{k}\left(t_{1}, \ldots, t_{2 n}\right)=\eta_{k}\left(t_{1}\right) \ldots \eta_{k}\left(t_{2 n}\right) .
$$

Then straightforward differentiation shows that for any $f \in I^{\infty}$, any mixed partial derivative of $\left(1-\xi_{k}\right) f$ of arbitrary order tends to zero uniformly on $\mathbf{R}^{2 n}$ as $k \rightarrow \infty$. By Proposition 4.10 of $[R]$, this means

$$
\lim _{k \rightarrow \infty}\left\|L_{\xi_{k} f}-L_{f}\right\|=0
$$

Hence $\left\{L_{f}: f \in \mathscr{S}\left(\mathbf{R}^{2 n}\right)\right\}$ is dense in $\left\{L_{f}: f \in I^{\infty}\right\}$ and $\pi_{J}\left(I_{J}\right)$ consists of the compact operators on $L^{2}\left(\mathbf{R}^{n}\right)$.

\section{Main Result}

We have, for Rieffel's algebra $A_{J}\left(\mathbf{R}^{2 n}\right)$ discussed in Sect. 3,

Theorem 2. The $C^{*}$-algebras $A_{J}\left(\mathbf{R}^{2 n}\right)$ and $\tau\left(\mathbf{B}_{2 n}\right)$ are $*$-isomorphic via $\operatorname{ad}_{V}^{-1} \circ \operatorname{ad}_{B} \circ$ $\pi_{J}$. Here, as usual, $\operatorname{ad}_{U}$ denotes the conjugation by $U$.

Proof. Recall that every element in $\tau\left(\mathbf{B}_{2 n}\right)$ is the sum of a Toeplitz operator whose symbol is continuous on $\mathbf{B}_{2 n}$ and a compact operator. We also recall that for $f$ in $C\left(S^{2 n-1}\right)$

$$
B^{-1} T_{\hat{f}} B=W_{\alpha_{f}}
$$


where $W_{\beta}$ was defined earlier as was the Bargmann isometry $B$ and

$$
\alpha_{f}(\xi, x)=\tilde{\hat{f}}(x-i \xi)
$$

We consider the $C^{*}$-algebra Weyl $\left(S^{2 n-1}\right)$ generated by the full algebra $\mathscr{K}$ of compact operators on $L^{2}\left(\mathbf{R}^{n}\right)$ and the operators

$$
\left\{W_{\alpha_{f}}: f \in C\left(S^{2 n-1}\right)\right\} .
$$

Using Theorem 1, it is not hard to check directly that conjugation by the unitary operator

$$
B^{-1} V: H^{2}\left(\mathbf{B}_{2 n}\right) \rightarrow L^{2}\left(\mathbf{R}^{n}\right)
$$

implements an isomorphism from $\operatorname{Weyl}\left(S^{2 n-1}\right)$ on $L^{2}\left(\mathbf{R}^{n}\right)$ onto $\tau\left(\mathbf{B}_{2 n}\right)$ on $H^{2}\left(\mathbf{B}_{2 n}\right)$.

In Sect. 3, we checked that Rieffel's algebra $A_{J}\left(\mathbf{R}^{2 n}\right)$ is $*$-isomorphic via $\pi_{J}$ to the $C^{*}$-algebra $\pi_{J}\left(A_{J}\left(\mathbf{R}^{2 n}\right)\right)$ on $L^{2}\left(\mathbf{R}^{n}\right)$ generated by the operators

$$
\left(\Psi_{T \gamma} g\right)(x)=(2 \pi)^{-n} \int_{\mathbf{R}^{n} \mathbf{R}^{n}}(T \gamma)(x+y, \quad \xi) e^{l(x-y)} \cdot \xi g(y) d y d \xi,
$$

where $\gamma$ is in $A^{\infty}$ with (uniform) radial limit function $\gamma_{\text {radial }}$ in $C\left(S^{2 n-1}\right)$. Moreover, comparing [R] and Sect. 3, $\pi\left(\Psi_{T \gamma}\right)=\gamma_{\text {radial }}$.

Because $\alpha_{f}$ is convolution of $\hat{f}$ with a Gaussian, it is easy to check that $T^{-1} \alpha_{f}$ is in $A^{\infty}$ of [R] for arbitrary $f$ in $C\left(S^{2 n-1}\right)$. This fact was already observed for $n=1$ in [R]. Using Eq. (7) of Sect. 3 and the fact that

$$
\left(\alpha_{f}\right)_{\text {radial }}(x, \xi)=f(\xi,-x),
$$

it is easy to check that $\operatorname{Weyl}\left(S^{2 n-1}\right)=\pi_{J}\left(A_{J}\left(\mathbf{R}^{2 n}\right)\right)$. Hence, $A_{J}\left(\mathbf{R}^{2 n}\right)$ and $\tau\left(\mathbf{B}_{2 n}\right)$ are *-isomorphic.

\section{Problems and Remarks}

It would be of some interest to know if other standard Toeplitz algebras arise as strict deformation quantizations of commutative algebras. In this connection, we should mention [BLU] where an "intrinsic" Toeplitz quantization on bounded symmetric domains is described following earlier work of [KL] and [C2]. The Toeplitz quantization of [KL, C2], [BLU] satisfies a weaker version of the strict deformation conditions required by $[\mathrm{R}]$.

Problem 1. Can the Toeplitz algebra on the polydisc, $\tau(\mathbf{D} \times \mathbf{D})$, be realized as a strict deformation quantization of $C(\mathbf{D} \times \mathbf{D})$,

While we have exhibited a $*$-isomorphism

$$
\mu: A_{J}\left(\mathbf{R}^{2 n}\right) \rightarrow \tau\left(\mathbf{B}_{2 n}\right)
$$

it is not obvious precisely what elements of $\tau\left(\mathbf{B}_{2 n}\right)$ are in $\mu\left\{A_{J}^{\infty}\left(\mathbf{R}^{2 n}\right)\right\}$.

Problem 2. Can $\mu\left\{A_{J}^{\infty}\left(\mathbf{R}^{2 n}\right)\right\}$ be precisely identified? 


\section{References}

[B] Bargmann, V.: On a Hilbert space of analytic functions and an associated integral transform. Commun. Pure and Appl. Math. 14, 187-214 (1961)

[BC1] Berger, C.A., Coburn, L.A.: Toeplitz operators and quantum mechanics. J. Funct. Anal. 68, 273-299 (1986)

[BC2] Berger, C.A., Coburn, L.A.: Toeplitz operators on the Segal-Bargmann space. Trans. Am. Math. Soc. 301, 813-829 (1987)

[BC3] Berger, C.A., Coburn, L.A.: Heat flow and Berezin-Toeplitz estimates. Am. J. Math. 116, 563-590 (1994)

[BDF] Brown, L.G., Douglas, R.G., Fillmore, P.A.: Extensions of $C^{*}$-algebras and $K$-homology. Ann. Math. 105, 265-324 (1977)

[BLU] Borthwick, D., Lesniewski, A., Upmeier, H.: Non-perturbative deformation quantization of Cartan domains. J. Funct. Anal. 113, 153-176 (1993)

[C1] Coburn, L.A.: Singular integral operators and Toeplitz operators on odd spheres. Indiana Univ. Math. J. 23, 433-439 (1973)

[C2] Coburn, L.A.: Deformation estimates for the Berezin-Toeplitz quantization. Commun. Math. Phys. 149, 415-424 (1992)

[F] Folland, G.B.: Harmonic analysis in phase space. Annals of Math. Studies. Princeton NJ, Princeton Univ. Press, 1989

[G] Guillemin, V.: Toeplitz operators in $n$-dimensions. Int. Eq. and Op. Thy. 7, 145-205 (1984)

[H] Howe, R.: Quantum mechanics and partial differential equations. J. Funct. Anal. 38, 188254 (1980)

[KL] Klimek, S., Lesniewski, A.: Quantum Riemann surfaces I, The unit disc. Commun. Math. Phys. 146, 103-122 (1992)

[R] Rieffel, M.A.: Deformation quantization for actions of $\mathbf{R}^{d}$. Memoirs of the Am. Math. Soc. 106, No. 506 (1993)

[S] Sheu, A.J.: Quantization of Poisson $S U(2)$ and its Poisson homogeneous space-the 2sphere. Commun. Math. Phys. 135, 217-232 (1991)

[V] Venugopalkrishna, U.: Fredholm operators associated with strongly pseudoconvex domains in $\mathbf{C}^{n}$. J. Funct. Anal. 9, 349-373 (1972)

[WW] Whittaker, E.T., Watson, G.N.: Modern Analysis. London: Cambridge Univ. Press, 1940

[X] Xia, J.: Geometric invariants of the quantum Hall effect. Commun. Math. Phys. 119, 29-50 (1988)

Communicated by A. Jaffe 